\title{
An Optimal Analysis in Wireless Powered Full-duplex Relaying Network
}

\author{
Kieu-Tam NGUYEN ${ }^{1}$, Dinh-Thuan DO ${ }^{1,2}$, Miroslav VOZNAK ${ }^{3}$ \\ ${ }^{1}$ Faculty of Electrical \& Electronics Engineering, Ton Duc Thang University, 19 Nguyen Huu Tho St., \\ 7th Dist., Ho Chi Minh City, Vietnam \\ ${ }^{2}$ Wireless Communications Research Group (WiCOM), Faculty of Electrical and Electronics Engineering, \\ Ton Duc Thang University, Ho Chi Minh City, Vietnam \\ ${ }^{3}$ VSB Technical University of Ostrava, 17. listopadu 15/2172, 70833 Ostrava - Poruba, The Czech Republic
}

\{nguyenkieutam,dodinhthuan\}@tdt.edu.vn, miroslav.voznak@vsb.cz

Submitted March 29, 2016 / Accepted September 11, 2016

\begin{abstract}
Wireless-powered cellular networks (WPCNs) are currently being investigated to exploit the reliability and improve battery lifetime of mobile users. This paper investigates the energy harvesting structure of the full-duplex relaying networks. By using the time switching based relaying (TSR) protocol and Amplify-and-Forward (AF) model in delay-limited transmission scheme, we propose the closedform expression of the outage probability and then calculate the optimal throughput. An important result can be taken obviously that the time fraction in TSR, the position of relay, the noise as well as the energy conversation impacting on the outage probability as well as the optimal throughput. By Monte Carlo simulation, the numerical results indicate an effective relaying strategy in full-duplex cooperative systems. Finally, we provide fundamental design guidelines for selecting time fraction in TSR that satisfies the requirements of a practical relaying system.
\end{abstract}

\section{Keywords}

Energy harvesting, full-duplex, one way relaying network, time switching-based protocol, throughput, amplify-and-forward

\section{Introduction}

The use of the regenerated energy in the next generation wireless systems such as $5 \mathrm{G}$ cellular network will bring about the considerable profits for the continuous operation in the wireless devices. Together with the other power resources such as solar, wind energy, the wireless power which are drawn from the radio-frequency (RF) signals departed by the surrounding transmitters can be replaced for the normal wired energy networks. Such energy harvested from the natural environment is a promising approach to maintain the life time of the energy constrained wireless nodes [1].
The authors in [2] considered simultaneous wireless power and information transfer (SWPIT) which is applied TS-based two-way relaying (TS-TWR) protocol. They investigated an optimization problem under total power constraint to examine the system throughput limit with data rate fairness.

In such that cases, a view of SWPIT networks with a special concentration on the hardware performance of the rectifying circuits and the real technics which get the SWPIT in the areas of the time, the space, the energy, the antennas as well as the profits of a potentiality synthesis of the SWPIT techniques in advanced information systems in the context of the source distributed and cooperative knowledge wireless systems are presented about in [3-5]. Moreover, a cognitive wireless network with one primary user (PU) and one secondary user (SU) and their transmitters run in TSR is considered. The SU, which collects the power solely from the surrounding wireless information, pursues a save-thentransmit protocol. In that way, a scenario, the SUs maximal cooperation strategic method, called, the maximal decision (in order to decide to work with the PU or not) and the maximal act (to consume how much time on the power collection and to distribute how much energy for the cooperative relay) is studied by the previous works as in [6-10].

On the other hand, an amplify-and-forward (AF) relaying system, in that, the power constrained relay node which collected the power from the receiving RF signal, utilized that collected power to transmit the resource communication to the target is studied. Relied on the time switching and power splitting receiving structures, two relaying protocols i) the time switching-based relaying (TSR) protocol and ii) the power splitting-based relaying (PSR) protocol are suggested, offer collecting the power and support the communication link to the relay. Pressed by model based on the canonical cognitive radio (CR) system form, an unusual way of the radio systems existing at the same time in which the short-energy proceduced in a second system, named, the sec- 
ondary transmitters (STs), collects the peripheral RF power from transmittances by the operation transceiver near around a main system, named, primary transmitters (PTs), whereas opportunely penetrating the spectrum authorized to the first system is suggested. A random-geometry pattern in that PTs and STs are allocated as the independence homogeneous Poisson point processes (HPPPs) and contacted with their planned transceivers at the unchanged spaces is examined in [11-17].

In fact, the authors in [18] indicated that the full-duplex mode is an attractive choice for the fixed relays provided that the loop interference power is maintained at a tolerable level. In addition, the authors in [19] presented the power allocation strategy to maximize the sum-rate of FD-two-way relaying system under the realistic residual self-interference (RSI).

Recently, a few research trends have been conducted in FD relay system in context of the SWIPT scheme. In [20], the throughput is analyzed for three relay control schemes, including the maximum relay, optimal relay, and target relay. Analytical expressions for the outage probability and ergodic capacity are also presented for these considered relay control schemes. However, no work related to the optimal throughput has considered for the application of the one-way FD relaying in RF energy harvesting systems.

Therefore, in this paper, we analyze the outage probability and the throughput of full-duplex relaying with the novel ability of the simultaneous energy harvesting and information transfer. Based on this analytical expressions, the best model for optimal energy efficiency are given.

Our contribution in this paper is to find out the optimal time fraction $\alpha_{\max }$ value to obtain maximum signal-to-noise ratio (SNR), from this result, we can give an optimal model for the energy harvesting full-duplex relaying network and prove that model satisfies for the optimal deployment in future cellular networks.

The remainder of this paper is organized as follows. Section 2 describes the system model of the EH enabled FD one-way relaying network. In Sec. 3 , the outage probability and throughput in delay-limited transmission mode are analysed. The simulation results are presented in Sec. 4. Finally, the conclusion of Sec. 5 is drawn in this paper.

\section{System Model}

Let us consider a wireless full-duplex relaying network with the AF protocol system illustrated as in Fig. 1, in which the destination node D can be received signal at long distance thanks to help of relay node. The system consists of three nodes, the resource node is denoted by $S$ and one relay node is denoted by $\mathrm{R}$. The relay node has two antennas, one of them is responsible for signal transmission and the other is responsible for the signal reception. The cooperative relay is assumed to be an energy constrained device so that it must harvest energy from the source, and then use harvested

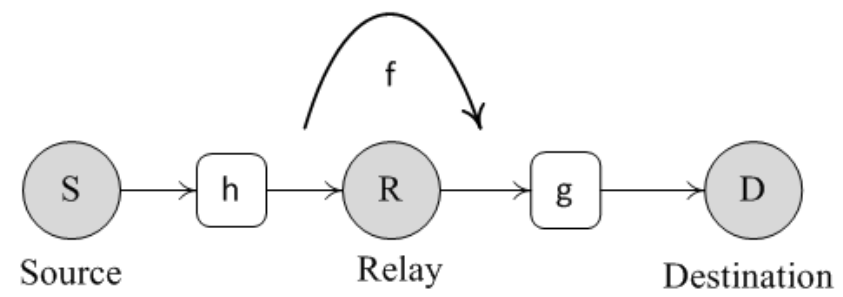

Fig. 1. System model of one way full-duplex relaying.

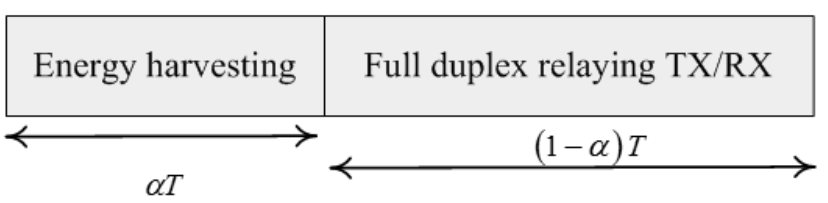

Fig. 2. Illustration of the parameters of TSR protocol.

energy to amplify and forward the source information to the destination node. We also assume that the direct link between source and destination node does not exist due to the deep shadowing effect or too long distance of direct link. We denote $h, g, f$ as channel at the first hop, the second hop and self-interference channel.

In this system model, the interference cancellation structure is adapted to eliminate the self-interference. Due to limit of circuits, the self-interference is unable to be cancelled entirely, a certain amount of its remains. The residual self-interference channel at $\mathrm{R}$ is defined by $f$. Let $d_{j}, j=1,2$ defined the gap of S-R link and R-D link, respectively and $h$, $g$ defined the channel coefficients of S-R link and R-D link, respectively. The model used in this study is the TSR protocol. The key parameters of protocol are depicted in Fig. 2.

Based on the TSR protocol, the information process is made into two stages. In the first stage, the energy is transferred from the source to the relay with a duration of $\alpha T$, $(0<\alpha<1)$ and in the second stage, the remaining time, $(1-\alpha) T$ is employed to transfer information, in which $\alpha$ is the time switching coefficient and $T$ is the time for the examined signal frame. In the energy harvesting phase, the received signal at the relay can be given by

$$
y_{\mathrm{R}}=\sqrt{\frac{P_{\mathrm{S}}}{d_{1}^{m}}} h x_{\mathrm{S}}+n_{\mathrm{R}}
$$

where $P_{\mathrm{S}}$ is the transmitted power from the source, $x_{\mathrm{S}}$ is transmit signal, $n_{\mathrm{R}}$ is the additive white Gaussian noise with zero-mean and variance of $\sigma_{n}^{2}$. Regarding the wireless received power, the harvested energy at the relay is given

$$
E_{h}=\Omega \alpha T \frac{P_{\mathrm{S}}|h|^{2}}{d_{1}^{m}}
$$

where $\Omega$ is energy conversion efficiency. In the information transfer stage, assuming that the source node transmits the respective signal $x_{\mathrm{S}}$ to $\mathrm{R}$ and $\mathrm{R}$ retransmits signal $x_{\mathrm{R}}$ to the destination node, $x_{j}, j=\mathrm{S}, \mathrm{R}$. It is assumed that the unit energy and zero mean, i.e, $E\left[\left|x_{j}\right|^{2}\right]=1$ and $E\left[x_{j}\right]=0$, in which $E[$.$] is expectation function. Therefore, the received$ 
signal at the relay under the self-interference constraint is given as

$$
y_{\mathrm{R}}=\sqrt{\frac{P_{\mathrm{S}}}{d_{1}^{m}}} x_{\mathrm{S}} h+f x_{\mathrm{R}}+n_{\mathrm{R}}
$$

where $x_{\mathrm{R}}$ is the transmit signal at $\mathrm{R}$. We suppose $\mathrm{R}$ receives $y_{\mathrm{R}}$ and in the next time slot, $\mathrm{R}$ uses that harvested energy to information processing for received signal $y_{R}$. Hence, using magnification of the prior received signal, $x_{\mathrm{R}}$, is illustrated as

$$
x_{\mathrm{R}}=K \sqrt{P_{\mathrm{R}}} y_{\mathrm{R}}
$$

where $K$ is the magnification coefficient of $\mathrm{R}$.

Based on principle of the AF relaying model at $R$, the magnification factor is computed by

$$
K=\left(\frac{P_{\mathrm{S}}}{d_{1}^{m}}|h|^{2}+P_{\mathrm{R}}|f|^{2}+\sigma_{n}^{2}\right)^{-1} .
$$

It is worth noting that the harvested power assists the operation for the next hop transfer, $P_{\mathrm{R}}$ is taken by

$$
P_{\mathrm{R}}=\frac{E_{h}}{(1-\alpha) T}=\varphi P_{\mathrm{S}} \frac{|h|^{2}}{d_{1}^{m}}
$$

where $\varphi$ is defined as $\varphi=\frac{\alpha \Omega}{1-\alpha}$.

Next, we get the received signal at destination as

$$
y_{\mathrm{D}}=\frac{g}{\sqrt{d_{2}^{m}}} x_{\mathrm{R}}+n_{\mathrm{D}}
$$

where $n_{\mathrm{D}}$ is Gaussian noise at destination. Substituting (4), (5), (6) into (7), we compute the receiving signal at destination node as

$$
\begin{aligned}
y_{\mathrm{D}} & =\underbrace{\left.P_{\mathrm{S}}|h|^{2}|| g\right|^{2}\left(|f|^{2}\right)^{-1}\left(d_{1}^{m}\right)^{-1}\left(d_{2}^{m}\right)^{-1}}_{\text {Signal }} \\
& +\underbrace{P_{\mathrm{S}}|h|^{2}\left(|f|^{2}\right)^{-1}\left(d_{1}^{m}\right)^{-1} \sigma^{2}\left(P_{\mathrm{R}}\right)^{-1}}_{R S I} \\
& +\underbrace{P_{\mathrm{R}}|g|^{2}\left(d_{2}^{m}\right)^{-1}+\sigma^{2}}_{\text {Noise }} .
\end{aligned}
$$

In the above equations, the instantaneous signal-tointerference-and-noise (SINR) at destination node is determined as

$$
\gamma_{\mathrm{D}}=\frac{E\left\{\mid \text { signal }\left.\right|^{2}\right\}}{E\left\{\mid \text { noise }\left.\right|^{2}\right\}+E\left\{|R S I|^{2}\right\}}
$$

By simple replacement, we get new formula as

$$
\begin{aligned}
\gamma_{\mathrm{D}} & =\frac{\gamma_{\mathrm{SD}} \Omega \alpha(1-\alpha)}{(1-\alpha)^{2}+\Omega^{2} \alpha^{2} \gamma_{\mathrm{LI}} \gamma_{\mathrm{SD}}+\sigma^{2} \eta \alpha \gamma_{\mathrm{LI}}(1-\alpha)} \\
& =\frac{a_{1} \alpha(1-\alpha)}{a_{2} \alpha^{2}+a_{3} \alpha+1}
\end{aligned}
$$

with

$$
\begin{aligned}
& a_{1}=\gamma_{\mathrm{SD}} \Omega \\
& a_{2}=1+\Omega^{2} \gamma_{\mathrm{LI}} \gamma_{\mathrm{SD}}-\sigma^{2} \Omega \gamma_{\mathrm{LI}} \\
& a_{3}=2+\sigma^{2} \Omega \gamma_{\mathrm{LI}}
\end{aligned}
$$

and

$$
\gamma_{\mathrm{SD}}=\frac{P_{\mathrm{S}}|h|^{2}|g|^{2}}{d_{1}^{m} d_{2}^{m} \sigma^{2}}=\operatorname{SIN}_{\mathrm{S}} \frac{|h|^{2}|g|^{2}}{d_{1}^{m} d_{2}^{m}}
$$

where $\operatorname{SIN}_{\mathrm{S}}=\frac{P_{\mathrm{S}}}{\sigma^{2}}, \gamma_{\mathrm{LI}}=\frac{|f|^{2}}{\sigma^{2}}$.

We find $\alpha$ with respecting maximal SNR, $\mathrm{SNR}_{\max }$, subject to $0<\alpha<1$, it is noted that $\gamma_{\mathrm{D}}(\alpha)$ is a concave function of the optimal value $\alpha$ which can be obtained by resolving the equation

$$
\frac{\partial \gamma_{\mathrm{D}}}{\partial \alpha}=0
$$

and after some manipulations, (13) results in following values

$$
\begin{aligned}
& \alpha_{1}=\frac{-1+\sqrt{1+a_{2}+a_{3}}}{a_{2}+a_{3}}, \\
& \alpha_{2}=\frac{-1-\sqrt{1+a_{2}+a_{3}}}{a_{2}+a_{3}}
\end{aligned}
$$

and there is only $\alpha_{1}$ satisfies the condition $0<\alpha<1$.

Replacing $\alpha_{1}$ into (10), we obtain new expression as

$$
\gamma_{\mathrm{D} \max }=\frac{C_{1} C_{2}}{A_{1}+B_{1}}
$$

where

$$
\begin{aligned}
& A_{1}=\left(3+\Omega^{2} \gamma_{\mathrm{LI}} \gamma_{\mathrm{SD}}\right) A_{2}, \\
& A_{2}=\left(1+\Omega^{2} \gamma_{\mathrm{LI}} \gamma_{\mathrm{SD}}-\sigma^{2} \Omega \gamma_{\mathrm{LI}}\right)\left(-1+\sqrt{3+\Omega^{2} \gamma_{\mathrm{LI}} \gamma_{\mathrm{SD}}}\right) \\
& B_{1}=\left(2+\sigma^{2} \Omega \gamma_{\mathrm{LI}}\right)\left(-1+\sqrt{3+\Omega^{2} \gamma_{\mathrm{LI}} \gamma_{\mathrm{SD}}}\right)+B_{2}, \\
& B_{2}=3+\Omega^{2} \gamma_{\mathrm{LI}} \gamma_{\mathrm{SD}}, \\
& C_{1}=\gamma_{\mathrm{SD}} \Omega\left(-1+\sqrt{3+\Omega^{2} \gamma_{\mathrm{LI}} \gamma_{\mathrm{SD}}}\right) \\
& C_{2}=4+\Omega^{2} \gamma_{\mathrm{LI}} \gamma_{\mathrm{SD}}-\sqrt{3+\Omega^{2} \gamma_{\mathrm{LI}} \gamma_{\mathrm{SD}}} .
\end{aligned}
$$

It is assumed that the channel gains $|h|^{2},|g|^{2}$ are independent and identically distributed (i.i.d.) exponential.

\section{Outage Probability and Throughput Analysis}

In this section, we derive the outage probability and the throughput of full-duplex one-way relaying with energy harvesting and information transfer. Based on that analytical expressions, the outage probability and the throughput of model are given and we can compare and evaluate them. Whereby, we can see that only time fraction parameter $\alpha_{\max }$ is been given the best performance in terms of maximal SNR and partial maximum throughput.

\subsection{Outage Probability Analysis} puted as

The outage probability of FD relaying network is com- 


$$
P_{\mathrm{o}}=\operatorname{Pr}\left(\gamma_{\mathrm{D}} \leq Z\right)
$$

where $R_{0}$ is target transmission rate and $Z=2^{R_{0}}-1$.

Equation (16) can be rewritten as follows

$$
\gamma_{\mathrm{D} \max }=\frac{D_{1} D_{3}\left(1-D_{2} D_{3}\right)}{D_{2}^{2}\left(D_{2}^{2}-D_{4}\right) D_{3}+D_{3} D_{4}+D_{2}^{2}}
$$

with

$$
\begin{aligned}
& D_{1}=\operatorname{SIN}_{\mathrm{S}}\left(\frac{|h|^{2}|g|^{2}}{d_{1}^{m} d_{2}^{m}}\right) \Omega, \\
& D_{2}=\sqrt{3+\Omega^{2} \gamma_{\mathrm{LI}} D_{1},} \\
& D_{3}=-1+D_{2}, \\
& D_{4}=2+\sigma^{2} \Omega \gamma_{\mathrm{LI}} .
\end{aligned}
$$

Proposition: The outage probability of the energy harvesting enabled one way AF full-duplex relay is given as

$$
\begin{aligned}
P_{\text {out }} & =\operatorname{Pr}\left\{\gamma_{\mathrm{D} \max } \leq Z\right\} \\
& =1-\int_{0}^{1 / \varphi Z} 2 \sqrt{\frac{d_{1}^{m} d_{2}^{m} Z\left(\frac{1}{\varphi}+y\right)}{\lambda_{h} \lambda_{g}\left(\operatorname{SIN}_{\mathrm{S}}-\varphi \operatorname{SIN} Z y\right)}} \\
& \times K_{1}\left(2 \sqrt{\frac{d_{1}^{m} d_{2}^{m} Z\left(\frac{1}{\varphi}+y\right)}{\lambda_{h} \lambda_{g}\left(\operatorname{SIN}_{\mathrm{S}}-\varphi \operatorname{SIN} N_{S} Z y\right)}}\right) \frac{1}{\lambda_{f}} \mathrm{e}^{-\frac{y}{\lambda_{f}}} \mathrm{~d} y
\end{aligned}
$$

where $\lambda_{h}, \lambda_{g}, \lambda_{f}$ are the mean value of the exponential random variables corresponding to $h, g, f$ channels, respectively and $K_{1}(x)$ is Bessel function denoted as (8.423.1) in [21].

Proof: We define $x=|h|^{2}|g|^{2}, y=\left|f_{\mathrm{R}}\right|^{2}$ and we have:

$$
P_{\mathrm{o}}= \begin{cases}\operatorname{Pr}\left\{x<\frac{d_{1}^{m} d_{2}^{m} Z\left(\frac{1}{\varphi}+y\right)}{\operatorname{SIN}_{\mathrm{S}}-\varphi \operatorname{SIN} Z \mathrm{~S} Z y}\right\}, & y<\frac{1}{\varphi \mathrm{Z}}, \\ 1, & y>\frac{1}{\varphi \mathrm{Z}} .\end{cases}
$$

Interestingly, the cumulative distribution function of $x$ is computed by

$$
\begin{aligned}
F_{x}(a) & =\operatorname{Pr}(x<a) \\
& =1-2 \sqrt{a / \lambda_{h} \lambda_{g}} K_{1}\left(2 \sqrt{a / \lambda_{h} \lambda_{g}}\right)
\end{aligned}
$$

and $y$ can be modeled with the probability distribution function $f_{y}(b)=\left(1 / \lambda_{f}\right) \mathrm{e}^{\left(b / \lambda_{f}\right)}$ and the proposition is attained after some simple calculation by hand.

On the other hand, following in [22], we have the DF outage probability given as

$$
\begin{aligned}
& P_{\mathrm{o}}^{\mathrm{DF}}= \\
& 1-\left(1-\mathrm{e}^{\frac{1}{\varphi \lambda_{r} \mathrm{Z}}}\right)\left(2 \sqrt{\frac{\sigma^{2} d_{1}^{m} d_{2}^{m} Z}{\varphi \lambda_{s} \lambda_{d} P_{\mathrm{S}}}}\right) K_{1}\left(2 \sqrt{\frac{\sigma^{2} d_{1}^{m} d_{2}^{m} Z}{\varphi \lambda_{s} \lambda_{d} P_{\mathrm{S}}}}\right) .
\end{aligned}
$$

\subsection{Optimal Throughput Analysis}

In the Proposition, the outage probability of the scheme, when the relay harvests the energy from the resource signal and employs that power to amplify and forward the resource signal to the destination, is a function of the energy harvesting time $\alpha$, and reciprocate together when $\alpha$ increases from 0 to 1 . In the delay-limited transmission protocol, the transmitter transfers signal at a fixed transmission rate $R_{0}$ (bits/sec/Hz) and $(1-\alpha) T$ is the affective information time. So, the throughput of network in delay-limited mode is expressed as

$$
\tau=\left(1-P_{\mathrm{o}}\right) R \frac{(1-\alpha) T}{T}
$$

We propose optimization problem for throughput in delay-limited transmission as

$$
\alpha^{*}=\underset{\text { s.t. } S N R \leq S N R_{\max }}{\arg \max } \tau .
$$

Unfortunately, it is hard to derive the optimal throughput mathematically due to integral included. However, this value can be obtained numerically by solving the equation $\frac{\mathrm{d} \tau}{\mathrm{d} \alpha}=0$. In addition, we can use either Golden section search method or gradient descent algorithm to find this optimal point. In the following numerical experiments, we find the optimal $\alpha$ based on the constraint of SNR and compare its value with different surrounding values.

\section{Numerical Results}

In this section, we use the derived analytical results to provide the outage probability, the optimal throughput, the optimal energy harvesting time. We set the source transmission rate $\mathrm{R}=3(\mathrm{bps} / \mathrm{Hz})$, and hence the outage SINR threshold is given by $Z=2^{\mathrm{R}}-1$. The energy harvesting efficiency is set to be $\Omega=0.4$ (eliminate Fig. 5, Fig. 6), $\sigma^{2}=0.1$ (exclude Figures 7-10, the path loss exponent is set to be $m=3$. For simplicity, we set the distance $d_{1}=d_{2}=1$ (exclude Fig. 3 and Fig. 4). Also, we set $\lambda_{h}=\lambda_{g}=1 ; \lambda_{f}=0.1$, unless in different special cases.

From Fig. 3, the outage probability of the different scenarios of time is allocated following $\alpha$ values. The outage probability is minimum at approximate $\alpha=\alpha_{\max }$ at high value of energy conversion efficiency, whenever $\alpha$ is as smaller than $\alpha_{\max }$, the higher the outage probability is. And the same thing happens when $\alpha>\alpha_{\max }$. In Fig. 4, it is clearly that the throughput of $\alpha \leq 0.1$ is better than that of $\alpha_{\max }$, let get together with above outage probability, of course, $\alpha_{\max }$ is the best value satisfy maximal SNR and remaining acceptable outage probability when we employ this model. As can be seen that the analysis curves provide a strictly agreement with simulation curves as in Fig. 3 and Fig. 4. 


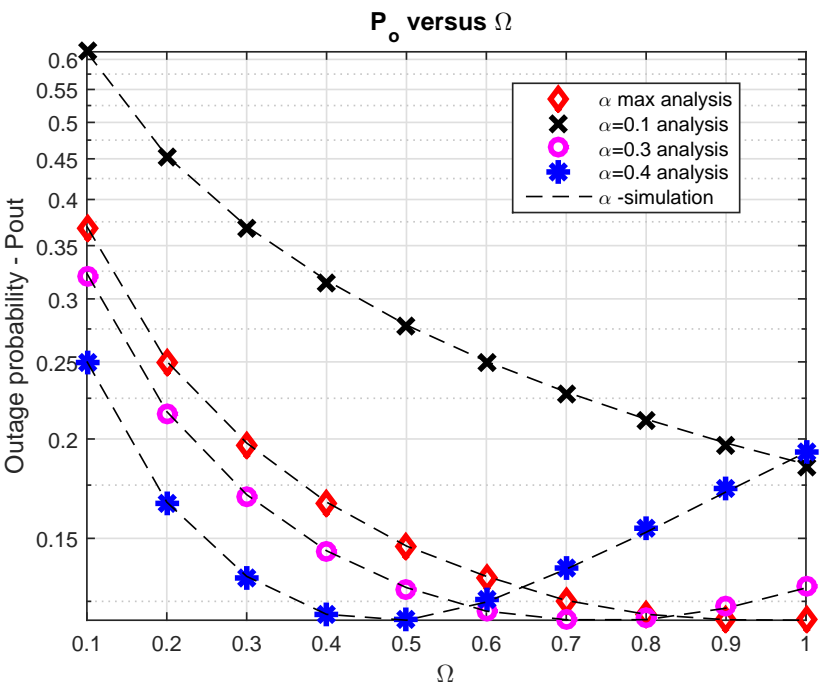

Fig. 3. Outage probability of FD energy-aware relaying network versus $\Omega$.

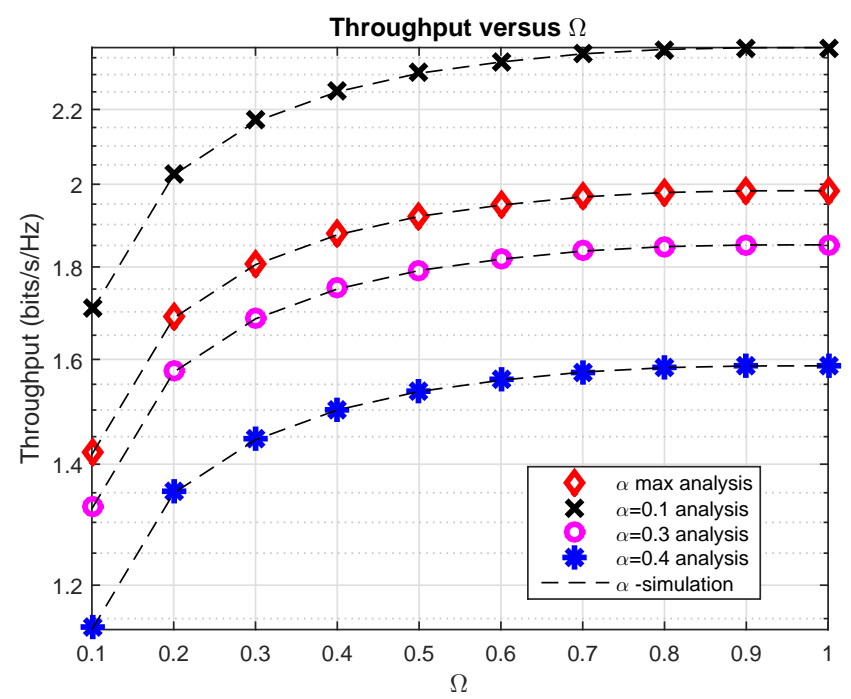

Fig. 4. Throughput of FD relaying versus $\Omega$.

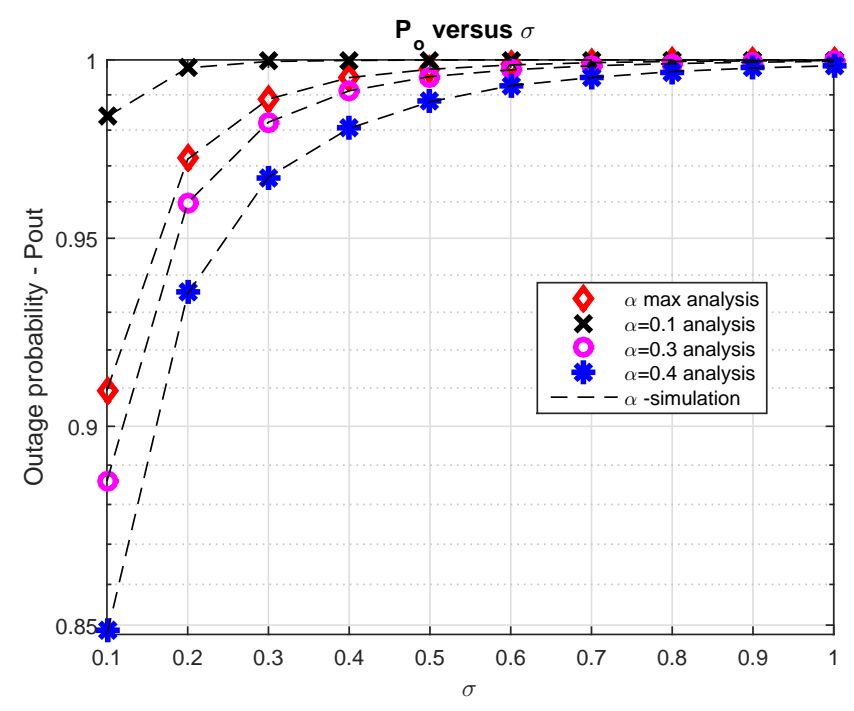

Fig. 5. Outage probability of FD relaying network versus noise term

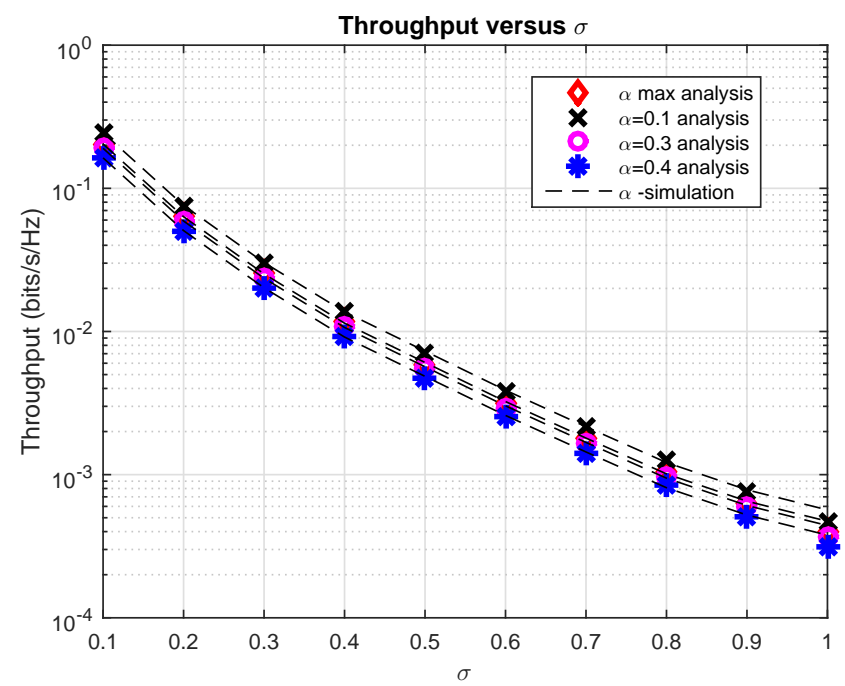

Fig. 6. Throughput of FD relaying versus noise term.

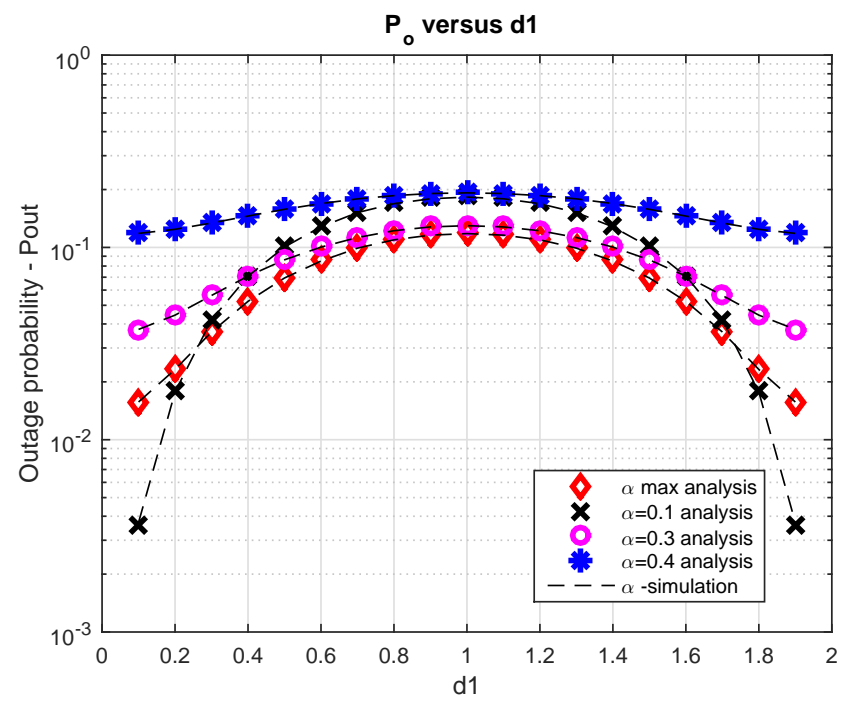

Fig. 7. Outage probability of FD relaying network versus $d_{1}$.

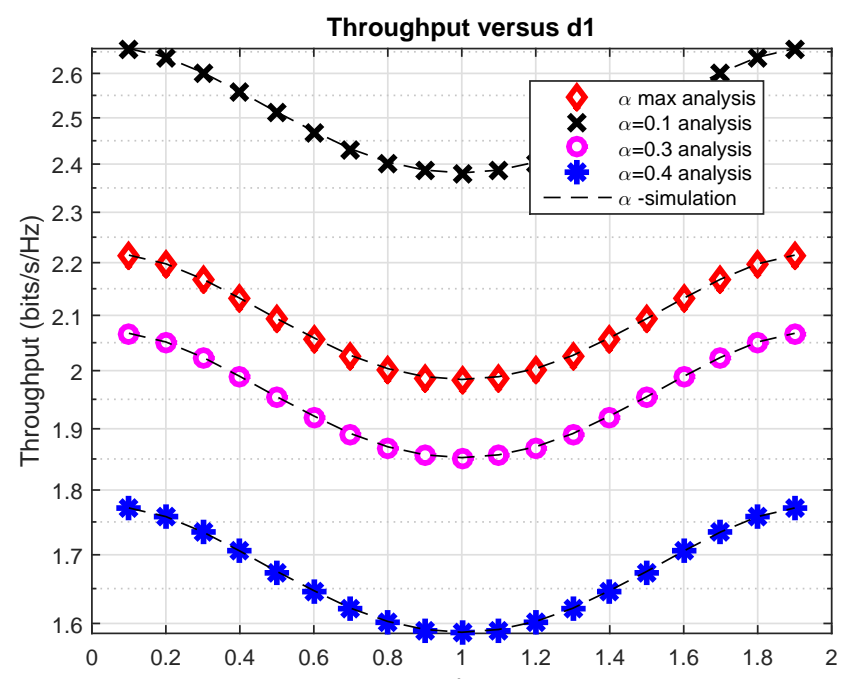

Fig. 8. Throughput of FD relaying versus $d_{1}$. 


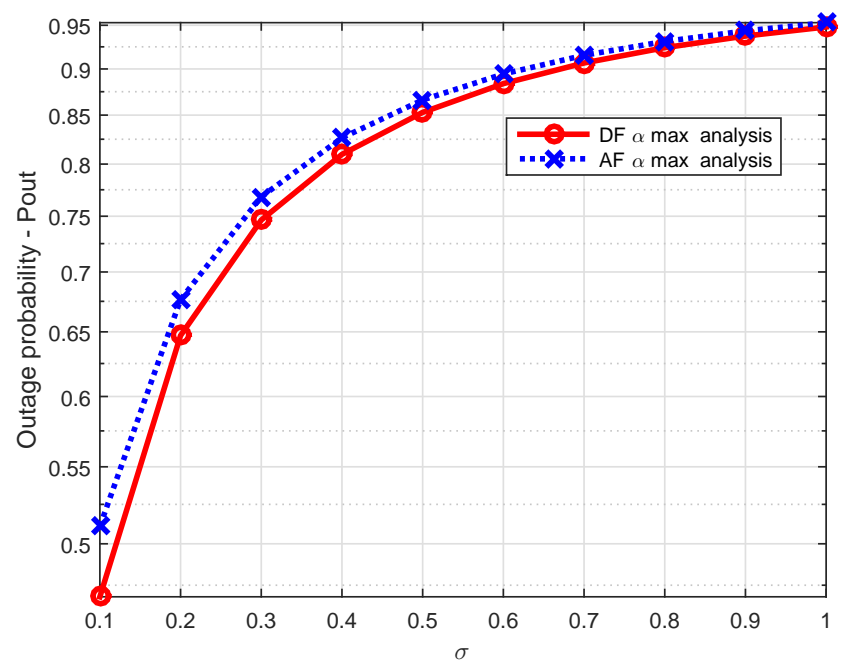

Fig. 9. Outage probability of AF and DF relaying versus $\sigma$.

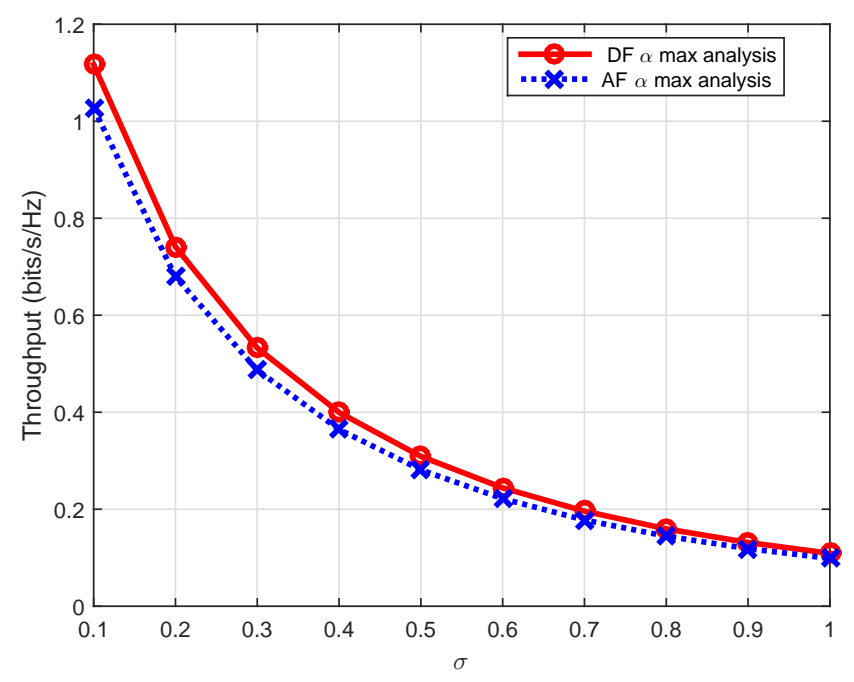

Fig. 10. Throughput of $\mathrm{AF}$ and $\mathrm{DF}$ relaying versus $\sigma$.

In Fig. 5, the smaller noise leads to the lower outage probability and this outage probability increases when $\sigma$ increases. The reason is that noise term will affect SNR and outage performance occurs, especially in high value of $\sigma$, i.e. $\sigma>0.6$. For the throughput, there is a different thing, throughput fall down remarkably in high noise range as illustration in Fig. 6.

Considering the impacts of distance on system performance in Fig. 7 and Fig. 8. It can be observed that the outage probability increases when $d_{1}$ increases from 0 to 1 and gets the worse outage values at the midway. While the closer at resource node, the smaller the outage probability can be obtained. This trend contraries to the throughput, so the relay location is much close to the resource node, the better performance we get. The same scenarios occur when we investigate the outage probability and the throughput with parameter $\sigma$, in Fig. 7 and Fig. 8 (the bigger $\sigma$, the bigger outage probability and the smaller throughput). At here, there is a little different thing, $\alpha$ is smaller or bigger than $\alpha_{\max }$, the bigger the outage probability is but when $\alpha \leq 0.1$, its throughput is better than that of $\alpha_{\max }$.
Next, the similar trend happens in Fig. 9 and Fig. 10, as bigger $\sigma$, as worse the outage probability of two model can be seen. Interestingly, the outage probability of DF model is better than that of AF model slightly and its throughput is better than, too. This is easily to explain because of noise at relay node which has impact on SNR and then results in system performance.

Remark 1: It worth noting that, there are two values of $\alpha$ lead to similar performance of outage and throughput, i.e. $\alpha_{\max }, \alpha=0.1$. In general, when comparing between optimal time switching $\alpha_{\max }$ and $\alpha=0.1$, it can be seen clearly that with $\alpha$ is smaller than $\alpha_{\max }$, its throughput is better than that of $\alpha_{\max }$, this is so easy to understand because when $\alpha$ is as smaller, as bigger the information quantity is sent to the destination but its outage probability is very high, it means that the operation of system may be stopped at higher level. And the second reason is that $\alpha$ is as smaller, the necessary energy which is harvested by the relay not enough power to transmit signal to the destination node. So, considering all of things, we find one thing that the model has the best performance for both maximal SNR and optimal throughput only with $\alpha_{\max }$.

\section{Conclusion}

In this paper, we have proposed a full-duplex relaying network with wireless energy harvesting and information transfer protocol, where an energy constrained relay node harvests energy from the received RF signal and uses that harvested the energy to forward the source signal to the destination in delay limited mode. In order to determine the achievable outage probability and throughput, the analytical expressions for the outage probability and throughput following the optimal value of the energy harvesting time in AF scheme of TSR protocol can be found by the simulation. As a result, we can see that the model deployed with $\alpha_{\text {max }}$ becomes the optimal model gotten the best performance.

\section{Acknowledgments}

This research is funded by Foundation for Science and Technology Development of Ton Duc Thang University (FOSTECT), website: http://fostect.tdt.edu.vn, under grant number FOSTECT.2016.BR21.

\section{References}

[1] ZIHAO, W., ZHIYONG, C., YAO, Y., et al. Wireless energy harvesting and information transfer in cognitive two-way relay networks. In Proceedings of the Global Communications Conference (GLOBECOM). Austin, TX (USA), 2014, p. 3465-3470. DOI: 10.1109/GLOCOM.2014.7037344

[2] KE, X., PINGYI, F., BEN LETAIEF, K. Time-switching based SWPIT for network-coded two-way relay transmission with data rate fairness. In Proceedings of the 2015 IEEE International Conference on Acoustics, Speech and Signal Process- 
ing (ICASSP). Queensland (Australia), 2015, p. 5535-5539. DOI: 10.1109/ICASSP.2015.7179030

[3] KE, X., PINGYI, F., CHUANG Z., et al. Wireless information and energy transfer for two-hop non-regenerative MIMO-OFDM relay networks. IEEE Journal on Selected Areas in Communications, 2015, vol. 33, no. 8, p. 1595-1611. DOI: 10.1109/JSAC.2015.2391931

[4] KRIKIDIS, I., TIMOTHEOU, S., NIKOLAOU, S., et al. Simultaneous wireless information and power transfer in modern communication systems. IEEE Communications Magazine, 2014, vol. 52, no. 11, p. 104-110. DOI: 10.1109/MCOM.2014.6957150

[5] LIU, Y., WANG, X. Information and energy cooperation in OFDM relaying: Protocols and optimization. IEEE Transactions on Vehicular Technology, 2015, vol. 65, no. 7, p. 5088-5098. DOI: 10.1109/TVT.2015.2455232

[6] MOUSAVIFAR, S. A., YUANWEI, L., LEUNG, C., et al. Wireless energy harvesting and spectrum sharing in cognitive radio. In Proceedings of the Vehicular Technology Conference (VTC Fall). Vancouver (Canada), 2014, p. 1-5. DOI: 10.1109/VTCFall.2014.6966232

[7] SIBOMANA, L., ZEPERNICK, H. J., HUNG, T. Wireless information and power transfer in an underlay cognitive radio network. In Proceedings of the 2014 8th International Conference on Signal Processing and Communication Systems (ICSPCS). Gold Coast, QLD (Australia), 2014, p. 1-7. DOI: 10.1109/ICSPCS.2014.7021107

[8] SIXING, Y., ERQING, Z., ZHAOWEI, Q., et al. Optimal cooperation strategy in cognitive radio systems with energy harvesting. IEEE Transactions on Wireless Communications, 2014, vol. 13, no. 9, p. 4693-4707. DOI: 10.1109/TWC.2014.2322972

[9] SIXING, Y., ZHAOWEI, Q., SHUFANG, L. Achievable throughput optimization in energy harvesting cognitive radio systems. IEEE Journal on Selected Areas in Communications, 2015, vol. 33, no. 3, p. 407-422. DOI: 10.1109/JSAC.2015.2391712

[10] EL SHAFIE, A. Space time coding for an energy harvesting cooperative secondary terminal. IEEE Communications Letters, 2014 vol. 18, no. 9, p. 1571-1574. DOI: 10.1109/LCOMM.2014.2337294

[11] SAKR, A. H., HOSSAIN, E. Cognitive and energy harvesting-based D2D communication in cellular networks: Stochastic geometry modeling and analysis. IEEE Transactions on Communications, 2015, vol. 63, no. 5, p. 1867-1880. DOI: 10.1109/TCOMM.2015.2411266

[12] NASIR, A. A., XIANGYUN, Z., DURRANI, S., et al. Relaying protocols for wireless energy harvesting and information processing. IEEE Transactions on Wireless Communications, 2013, vol. 12, no. 7 , p. 3622-3636. DOI: 10.1109/TWC.2013.062413.122042

[13] DO, D.-T. Energy-aware two-way relaying networks under imperfect hardware: Optimal throughput design and analysis. Telecommunication Systems, 2015, vol. 62, no. 2, p. 449-459. DOI: 10.1007/s11235015-0085-7

[14] YANG, H., CLERCKX, B. Joint wireless information and power transfer for an autonomous multiple antenna relay system. IEEE Communications Letters, 2015, vol. 19, no. 7, p. 1113-1116. DOI: 10.1109/LCOMM.2015.2428252

[15] DO, D.-T. Power switching protocol for two-way relaying network under hardware impairments. Radioengineering, 2015, vol. 24 , no. 3 , p. 765-771. DOI: $10.13164 /$ re.2015.0765

[16] AHMED, I., IKHLEF, A., SCHOBER, R., et al. Joint power allocation and relay selection in energy harvesting AF relay systems. IEEE Wireless Communications Letters, 2013, vol. 2, no. 2, p. 239-242. DOI: 10.1109/WCL.2013.012513.130007
[17] HONG, X., KAI-KIT, W., NALLANATHAN, A. Secure wireless energy harvesting-enabled AF-relaying SWIPT networks. In Proceedings of the IEEE International Conference on Communications (ICC). London (UK), 2015, p. 2307-2312. DOI: $10.1109 /$ ICC.2015.7248669

[18] RIIHONEN, T., WERNER, S., WICHMAN, R. Comparison of fullduplex and half-duplex modes with a fixed amplify-and-forward relay. In Proceedings of the 2009 IEEE Wireless Communications and Networking Conference (WCNC). Budapest (Hungary), 2009, p. 1-5. DOI: $10.1109 / \mathrm{WCNC} .2009 .4917634$

[19] YANG, J., LIU, X., YANG, Q. Power allocation of two-way fullduplex AF relay under residual self-interference. In Proceedings of the 2014 IEEE 14th International Symposium on Communications and Information Technologies (ISCIT). Incheon, (South Korea), p. 213217. DOI: 10.1109/ISCIT.2014.7011903

[20] ZHONG, C., SURAWEERA, H. A., ZHENG, G., et al. Wireless information and energy transfer with full-duplex relaying. IEEE Transactions on Communications, 2014, vol. 62, no. 10, p. 3447-3461. DOI: 10.1109/TCOMM.2014.2357423

[21] DAVID, H. A. Order Statistics. 1st ed. New York, NY (USA): Wiley, 1970.

[22] NGUYEN, K. T., NGUYEN, H. T., NGUYEN, T.-Q., et al. A performance analysis in energy harvesting full-duplex relay. In Proceedings of the 1st International Conference on Applied Mathematics in Engineering and Reliability. Ho Chi Minh City (Vietnam), May 2016, p. 139-143. DOI: 10.1201/b21348-23

\section{About the Authors ...}

Kieu-Tam NGUYEN was born in Phu Yen Province, Vietnam. He received his M.Sc. from University of Transport and Communications, Viet Nam in 2012. Now, he is a Ph.D. student at VSB-Technical University of Ostrava, Czech Republic. His research interests include the wireless communication, WiMax and energy harvesting.

Dinh-Thuan DO (corresponding author) was born in Phu Yen Province, Vietnam. He received his Ph.D. from University of Science (VNUHCM) in 2012. Dr. Thuan was the recipient of the 2015 Golden Globe Award by Ministry of Science and Technology. He is currently Assistant Professor at the Wireless Communications Lab (WICOM LAB). His research interests include Wireless channel modelling and simulation, Massive MIMO, Cooperative MIMO communications, Energy harvesting, Full-duplex communications, Cognitive radio.

Miroslav VOZNAK is Associate Professor, VSB-Technical University of Ostrava, Czech Republic. His research interests include Wireless communications, Next generation networks, IP telephony, Security and Speech quality, 5G wireless communication networks. 